\title{
Evaluation of Signal and Noise and \\ Identification of a Suitable Target Function in the Tuning of an ESI Ion Trap Mass Spectrometer by Multivariate Pattern Recognition Tools
}

\author{
Emilio Marengo, Elisa Robotti, Fabio Gosetti, Orfeo Zerbinati, \\ and Maria Carla Gennaro \\ Department of Environmental and Life Sciences, University of Eastern Piedmont, Alessandria, Italy
}

When mass spectrometry is not combined to separation techniques, the evaluation of signal and noise in a complex mass spectrum is not trivial. The tuning of the spectrometer based only on the increase of the signal of a selected number of $\mathrm{m} / \mathrm{z}$ values does not ensure the achievement of the best experimental conditions: signal could improve and noise could increase as well. The scope of this work is the development of a function separating signal and noise (for evaluating the $\mathrm{S} / \mathrm{N}$ ) from complex mass spectra for potential use as target function for the automatic tuning of the instrument. Two different methods were applied: the first is based on the separation of a pool of $\mathrm{m} / \mathrm{z}$ values attributable to the signal from the $\mathrm{m} / \mathrm{z}$ values due to the noise, while the second is based on the application of principal component analysis to separate the signal (present in the significant components) from the noise (present in the residuals). The comparison of the two methods was carried out by the evaluation of the stability of the signal and the target functions obtained, and the evaluation of the variation of the target functions as a function of concentration. (J Am Soc Mass Spectrom 2009, 20, 1859-1867) (C) 2009 Published by Elsevier Inc. on behalf of American Society for Mass Spectrometry

$\mathrm{M}$ ass spectrometry (MS) is one of the most exploited analytical tools above all if coupled to liquid chromatography [1-4]. In particular, the use of an electrospray ion source (ESI) coupled to an ion trap (IT) analyzer provides increasing selectivity [1-4]. Mass spectrometry characterized by an ESI IT system is usually exploited for qualitative and quantitative analyses coupled to high-performance liquid chromatography (HPLC): the combination of these two techniques makes it possible the evaluation of quality parameters of the analytical method (e.g., signal-to-noise $(\mathrm{S} / \mathrm{N})$ ratio, limits of detection, and quantification) through the analysis of the chromatogram obtained. Mass spectrometry is seldom used by itself for quantitative analysis (in this case $\mathrm{MS}^{n}$ experiments are usually carried out) and in literature it is not possible to find general methods for evaluating $\mathrm{S} / \mathrm{N}$ and other quality parameters directly from complex mass spectra. The evaluation of signal and noise in a complex mass spectrum is not trivial and usually $\mathrm{MS}^{n}$ experiments are used to get around the problem. The possibility of separating the contribution of signal and noise and,

Address reprint requests to Professor E. Marengo, University of Piemonte Orientale, DISAV Dipartimento di Scienze dell'Ambiente e della Vita, Viale Michel 11, 15121 Alessandria, Italy. E-mail: marengoe@tin.it therefore, evaluating the $\mathrm{S} / \mathrm{N}$, is of fundamental importance from the point of view of quantitative analysis: it allows a first evaluation of limits of detection and quantification, and provides a suitable target function for optimization of the experimental parameters. This last application is very important since the mass spectrometer has to undergo a process of tuning, due to the large number of instrumental parameters, related to both ion source and analyzer that have to be set before MS experiments. In fact, the tuning of the spectrometer based only on the increase of the signal of a selected number of $\mathrm{m} / \mathrm{z}$ values does not ensure the achievement of the best experimental conditions, since also noise could increase.

The present work has two aims: (1) the identification of a method of general validity to evaluate signal and noise (and provide an evaluation of $\mathrm{S} / \mathrm{N}$ ) in complex mass spectra; (2) the development of a suitable target function for the automatic tuning for a future optimization of the instrumental parameters.

In general, the automatic tuning exploits a procedure that operates small changes to each experimental parameter to improve the signal of a selected number of $\mathrm{m} / \mathrm{z}$ values: the channels can change according to the standard mixture suggested by the manufacturer or to the choice of standard mixtures suitable for covering 
the overall $m / z$ range [5-8]. In all cases, whatever single channels or pools of $m / z$ values are selected, the tuning procedure searches for experimental settings providing the overall increase of intensity of the selected channels, while no consideration is given to the concurrent increase in noise. The default conditions adopted in this study were suggested by the manufacturer and involved the use of a complex calibration standard mixture constituted by caffeine, a tetra peptide, and Ultramark 1621 (Pelham, NH, USA) characterized by a pattern of $\mathrm{m} / \mathrm{z}$ values covering the overall $\mathrm{m} / \mathrm{z}$ range available by the mass analyzer $(50-2000 \mathrm{~m} / \mathrm{z})$. The standard mixture is characterized by high concentration and long persistence $[9,10]$. The actual automatic tuning procedure applied by the software in the calibration makes use of the signal recorded for $5 \mathrm{~m} / \mathrm{z}$ values covering the overall range of $\mathrm{m} / \mathrm{z}$ available $(195,524$, $1222,1522,1822 \mathrm{~m} / \mathrm{z}$ ); while the improvement of the intensities of the selected $\mathrm{m} / \mathrm{z}$ values is reached, there is no control on the possible contemporary increase of noise. The same problem is met with other automatic procedures reported in literature [5-8]. From these starting considerations, we applied two different methods for the evaluation of signal and noise and for the development of a suitable target function: (1) the first method, similar to that applied by standard automatic tuning, is based on the separation of a pool of $\mathrm{m} / \mathrm{z}$ values ascribed to signal, from other channels due to noise; (2) the second method is based on the application of principal component analysis (PCA) to separate the signal (present in the significant principal components) from the noise (present in the residuals) [11, 12].

Both methods allow the automatic identification of signal and noise from a pool of full scan mass spectra recorded by the instrument used, but they can be of general validity and could represent automatic tools to be implemented directly in any instrument software. Preliminary considerations based on the analysis of autocorrelation of subsequent scans showed the necessity to implement a method robust to the presence of trends and drifts in the instrumental response.

In the first method, similar to the one applied by the manufacturer, the $\mathrm{m} / \mathrm{z}$ channels containing the signal are automatically separated from those containing only noise to evaluate $\mathrm{S} / \mathrm{N}$. This method could shed light on the effectiveness of standard automatic procedures since it represents a generalization of the tuning methods currently applied by manufacturer based only on the increase of intensity of a selected pool of $\mathrm{m} / \mathrm{z}$ values.

The second method is based on a multivariate approach, as PCA, and separates the intensity recorded for each $\mathrm{m} / \mathrm{z}$ value in two contributions: one due to the signal and one to the noise. Both methods allow the identification of two mass spectra, describing the signal and the noise, respectively, and allowing the calculation of the S/N parameter. They are both applied, and stability and reliability are compared.

To this purpose, the following comparison was carried out: (1) evaluation of the stability of the signal and of the target functions in four replicates of a series of subsequent scans recorded for the standard mixture; (2) evaluation of the variation of the target functions with concentration in a sequence of scans recorded on the standard mixture solutions at decreasing and increasing concentration. This point is extremely important since the target function identified has to maintain correspondence with concentration, assuring once again that any difference in the signal is not related to comparable differences in the noise.

\section{Theory}

\section{Autocorrelation Function}

When data are obtained in sequence, measurements close in time are often more similar. The autocorrelation function provides a way of evaluating the linear dependence of successive measurements according to a selected time interval between the observations, the lag [11, 12]. Evaluating the autocorrelation between each observation and the preceding one corresponds to lag $=1$. The autocorrelation coefficient $r_{k}$ at lag $k$ of a series of $n$ measurements $y_{i}(i=1, \ldots n)$ is calculated as:

$$
r_{k}=\frac{\sum_{i=1, n-k}\left[\left(y_{i}-\bar{y}\right) \cdot\left(y_{i+k}-\bar{y}\right)\right]}{\sum_{i=1, n}\left(y_{i}-\bar{y}\right)^{2}}
$$

where:

$$
\begin{aligned}
& y_{i}=\text { the } i \text {-th measurement } \\
& \bar{y}=\text { the average value of all measurements } \\
& y_{i+k}=\text { measurement at time } i+k
\end{aligned}
$$

The coefficient $r_{k}$ can assume values ranging from -1 to +1 : the sign indicates positive or negative linear correlation between observations at lag $k$; a coefficient close to \pm 1 indicates a large dependence of measurement along time (according to the selected lag), while values around zero correspond to linearly independent observations.

In this case, the autocorrelation was evaluated with lag $=1$ since our aim was the identification of dependencies between two subsequent observations.

\section{Principal Component Analysis (PCA)}

PCA is a multivariate pattern recognition method allowing the representation of the original dataset in a new reference system described by new variables, called principal components (PCs) [11, 12]. PCs, linear combinations of the original variables, are orthogonal, hierarchically ranked, and can be used for an effective dimensionality reduction by considering only the first PCs that contain the systematic, relevant information (the last ones contain experimental error and random variations). The coordinates of the samples in the new reference system are called scores, while the weights of the original variables on each PC are called loadings. 


\section{The Procedure}

The two methods based on the: (1) separation of a pool of $\mathrm{m} / \mathrm{z}$ values due to signal from the other $\mathrm{m} / \mathrm{z}$ values due to noise and (2) application of PCA to separate signal (present in the significant principal components) from noise (present in the residuals), were evaluated and compared by a five steps procedure:

\section{Experimental Evaluation of Instrumental Stability}

Four genuine replicates of a series of 1000 subsequent scans are carried out on the standard mixture. These measurements are used both for the evaluation of the signal stability and of the number of scans suitable to obtain a stable signal.

\section{Evaluation of Experimental Response Modification with Varying Concentration}

A sequence at decreasing and increasing concentration of the standard mixture is recorded; 1000 scans are recorded for each of three concentration levels in sequence without interrupting MS scan. These measurements are used to evaluate if the target functions calculated provide a response properly varying with concentration. The 1000 scans are used to provide information about the stability of the instrumental response and are not necessary during common lab practice.

\section{Preliminary Considerations}

The autocorrelation function is calculated for each series of 1000 scans to check the independence of subsequent intensities recorded for each $\mathrm{m} / \mathrm{z}$ value. The evaluation of autocorrelation appears fundamental since the signal recorded by the instrument in subsequent scans should not show dependency; memory and saturation effects should not be present. Therefore, unstable autocorrelation values not centered around zero indicate an unstable instrumental functioning that has to be monitored and controlled.

\section{Application of the First Method}

This method allows the automatic separation of the $\mathrm{m} / \mathrm{z}$ values of full scan mass spectra in those due to signal and noise. Two parameters are varied during the analysis: the number of scans and channels that are considered signal as a function of a selected number of scans. This analysis was previously carried out on the replicates of 1000 scans recorded at the same concentration level and afterwards on the sequence recorded at different concentration levels. For each pair of parameters, two spectra are obtained: one of the signal, and one of the noise, showing complementary channels (if a $\mathrm{m} / \mathrm{z}$ value is included in the first spectrum, it is excluded from the second). The final index, calculated as the sum (corresponding to a total ion current-TIC) of both signal and noise spectra, is then analyzed to check whether it is suitable as target function. This method represents a generalization and automatization of the different procedures currently adopted for standard tuning; it is used here to investigate whether such procedures can provide an evaluation of $\mathrm{S} / \mathrm{N}$ parameter stable and varying properly with concentration, avoiding the contemporary increase of both signal and noise.

\section{Application of the Second Method}

The second method is based on the use of PCA; it can in facts separate the contribution due to signal from that due to noise. If PCA is carried out on the data matrix constituted by the subsequent 1000 scans at the same concentration level of the standard mixture (described by the $m / z$ values from 50 to 2000), without centering or scaling the data, the first PC aligns along the average signal, while noise is accounted for by the residuals. The intensity recorded for each channel is therefore separated into two contributions: one due to signal (accounted for by the first PC) and one due to noise (accounted for by the residuals). PCA is then first applied to three replicates of 1000 scans each at the same concentration level to analyze the stability of the target function (the first PC) calculated. Then, PCA is applied to the sequences recorded at different concentration levels of the standard solution, to study the variation of the target function with concentration.

\section{Analysis of the Results}

The results obtained by the two methods are then compared, and the one providing an estimation of $\mathrm{S} / \mathrm{N}$ parameter more stable and varying properly with concentration is then selected. The multivariate method is compared with the first method, pointing out advantages and disadvantages of both procedures.

\section{Experimental}

\section{Apparatus}

Mass spectrometry experiments were conducted by means of Thermoquest LCQ Duo ion trap mass spectrometer from Finnigan (San Jose, CA, USA) equipped with electrospray ionization (ESI). The MS software used was Xcalibur 2.0 SR2 (ThermoElectron Corporation, San Jose, CA, USA). Statistical calculations and graphical representations were carried out by Excel 2003 (Microsoft Corporation, Redmount, WA, USA), The Unscrambler ver. 9.2 (Camo, Oslo, Norway) and home-made algorithm developed in Matlab R2007b (The Mathworks, Natick, MA, USA). 


\section{Reagents}

Ultrapure water was produced by a Millipore Milli-Q system (Milford, MA, USA). Methanol CHROMASOLV (>99.9\%) and caffeine $(99 \%)$ are acquired from SigmaAldrich (Milwaukee, WI, USA). HPLC grade acetonitrile were acquired from Merck (Darmstadt, Germany). MRFA (methionine-arginine-phenylalanine-alanine) peptide mass spectrometry standard was purchased from Research Plus (Manasquan, NJ, USA) and Ultramark 1621 mass spectrometry standard from Lancaster Synthesis (Pelham, NH, USA). Glacial acetic acid for LC-MS was acquired from Fluka (Buchs, Switzerland).

\section{Preparation of Calibration Solution}

For calibrating the instrument, a solution of caffeine, MRFA, and Ultramark 1621 in a mixture solvent (acetonitrile, methanol, water) containing $1 \%$ of acetic acid must be used as requested by the manufacturer.

A calibration solution of $5.0 \mathrm{~mL}$ was prepared by mixing $100.0 \mu \mathrm{L}$ of caffeine methanol solution $(1 \mathrm{mg}$ $\left.\mathrm{mL}^{-1}\right), 5.0 \mu \mathrm{L}$ of MRFA methanol/water $50 / 50 \mathrm{vol} / \mathrm{vol}$ solution ( $\left.5 \mathrm{nmol} \mu \mathrm{L}^{-1}\right)$, and $2500 \mathrm{~mL}$ of Ultramark 1621 acetonitrile solution $(0.1 \% \mathrm{vol} / \mathrm{vol})$ with $50.0 \mu \mathrm{L}$ of glacial acetic acid and $2.340 \mathrm{~mL}$ of $50 / 50$ methanol/ water solution. The calibration solution was preserved in the dark in the refrigerator at $4{ }^{\circ} \mathrm{C}$, and remained stable for about 2 mo.

\section{Mass Spectrometry Conditions}

High purity nitrogen was used as nebulizer (sheath gas pressures set at 20 of the arbitrary scale $0-100$ of the instrument) and helium (>99.999\%) served as the quenching agent. The ESI probe tip and capillary potential were set at $5.00 \mathrm{kV}$ and $21.00 \mathrm{~V}$, respectively. The heated capillary was set at $200.00{ }^{\circ} \mathrm{C}$, and the ion optics parameters were set as follows: tube lens offset $0.00 \mathrm{~V}$, first octopole voltage $-2.25 \mathrm{~V}$, inter octopole lens voltage $-30.00 \mathrm{~V}$, second octopole voltage $-7.50 \mathrm{~V}$. AGC value was set automatically. Full scan data acquisition was made in positive ion mode over the range $\mathrm{m} / \mathrm{z}$ $50-2000$. The maximum ionization time is set at $200 \mathrm{~ms}$ with 3 microscans for scan. ESI mass spectra profiles are obtained by direct infusion (flow rate $5.0 \mu \mathrm{L} \mathrm{min}^{-1}$ ) of calibration solution recording 1000 scans (a typical full scan mass spectrum recorded is reported as Supplementary Material, which can be found in the electronic version of this article).

Four replicates were carried out at different time points during the day and on different days. The four replicates were run on four random days.

Then, a sequence was recorded by direct infusion of the calibration solution at decreasing and increasing concentration levels. Scan was not interrupted along the sequence. The sequence was recorded with variable concentration levels in this order: not diluted, diluted 1:10 vol/vol, 1:100 vol/vol, 1:10 vol/vol, not diluted.
The dilution was made by the solvent used for calibration. For each concentration level, a total of 1000 scans were recorded.

\section{Results and Discussion}

\section{Preliminary Considerations}

Some preliminary considerations were made about stability and presence of trends and drifts of the intensity recorded at each $\mathrm{m} / \mathrm{z}$ value in full scan analyses. The autocorrelation function ( $\operatorname{lag}=1$ ) was calculated for each replicate of 1000 scans at the same concentration level. The autocorrelation, calculated for each channel registered and for each pair of subsequent scans, indicates how each scan is related to the previous one. The autocorrelation function assumes particular interest here since it might represent a parameter related to good instrumental functioning; large autocorrelation between subsequent scans indicates the presence of memory or saturation effects that should not be present. Automatic calculation of the autocorrelation function could therefore provide an estimate of independence between subsequent measurements.

To analyze long-term instrumental effects, the four replicates were run on different days at different times during the day (morning or afternoon).

The $r_{1}$ values calculated are large positive in two replicates, at intermediate values in one case and quite small in the other case (the corresponding surface plots are provided as Supplementary Material). Autocorrelation seems very unstable, and the behavior is in general similar for all the $\mathrm{m} / \mathrm{z}$ values. This result is quite unexpected since experimental spectra obtained in subsequent measurements should be independent if no memory effect is present. This trend is not easily detectable by simply looking at subsequent spectra recorded, and the $r_{1}$ parameter could represent an effective index to evaluate this behavior.

The autocorrelation function was calculated also for the sequence recorded at different concentration levels (surface plot reported as Supplementary Material Figure 2e) to check whether it is independent from the concentration of the standard; for the first levels, the $r_{1}$ function is largely positive for all the $\mathrm{m} / \mathrm{z}$ values registered, then, from a certain point on, it drops almost to zero. The instrument seems to work in two different conditions, i.e., two different settings causing a sudden change of its response.

The stability is not directly related to the concentration level since the $r$ function drops after about 300 scans at the lowest concentration level and does not vary along with the concentration level. The sudden change of the experimental conditions could probably be due to instrumental factors or to saturation effects taking place in the ion trap (even if the AGC function should prevent such saturation effect).

The behavior pointed out certainly represents a problem, since it is not dependable from the moment 
during the day when measurements were recorded (morning or afternoon) or from the concentration level; there seems to be a sudden and unpredictable change. In these conditions, many difficulties arise during the identification of a univocal target function and also during the tuning of the instrument; it is not possible to ascribe changes in signal intensity to a sudden change in the instrumental response or to the tuning working out. This aspect is even more astonishing since the way automatic tuning is actually carried out does not take it into proper consideration. This is true also for other automatic procedures currently adopted by different manufacturers. It is mandatory to establish a procedure to highlight the sudden change in the behavior of the instrumental response, and then take it into account or solve it. From this point of view, the autocorrelation function could be successfully used as an index of good instrumental functioning.

From these considerations emerges the importance of establishing a target function robust to the presence of such discontinuities, and exploiting a method for signal and noise identification that is able to consider this effect.

First Method: Separation of $\mathrm{m} / \mathrm{z}$ Values Due to Signal and Noise

This method represents a generalization of different procedures present in literature where automatic tuning is commonly carried out by increasing the signal of a single or a pool of selected channels. It allows the general evaluation of the performance of automatic tuning procedures since it is mainly based on the automatic separation between $\mathrm{m} / \mathrm{z}$ values due to signal and noise. Two parameters were studied: number of scans and number of channels considered signal as a function of a selected percentage of scans. First of all, the effect of the variation of the two parameters was studied on the replicates of 1000 scans recorded at the same concentration level: for each $m / z$, if the intensity recorded is different from zero for more than a fixed
1000 scans, $100 \%$

Signal spectrum: $78 \mathrm{~m} / \mathrm{z}$ values

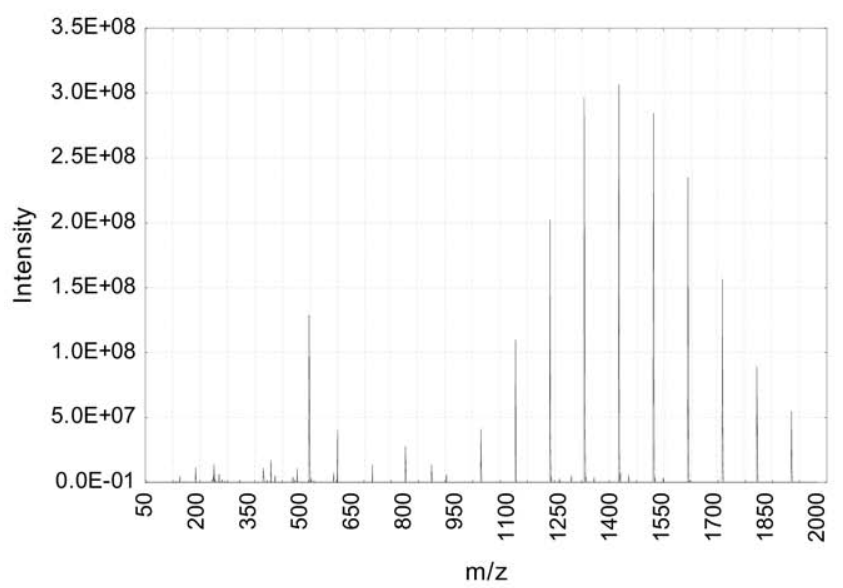

Noise spectrum: $1873 \mathrm{~m} / \mathrm{z}$ values

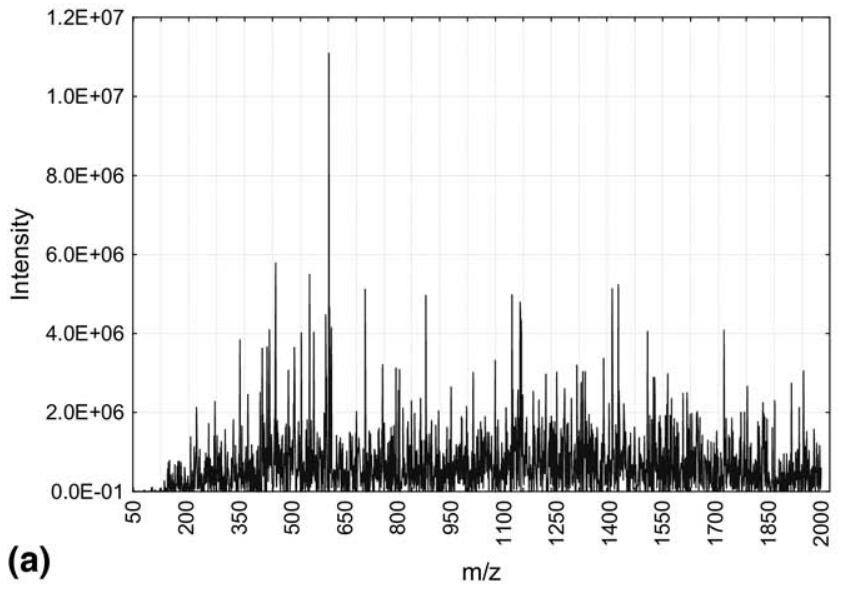

1000 scans, $85 \%$

Signal spectrum: $1743 \mathrm{~m} / \mathrm{z}$ values

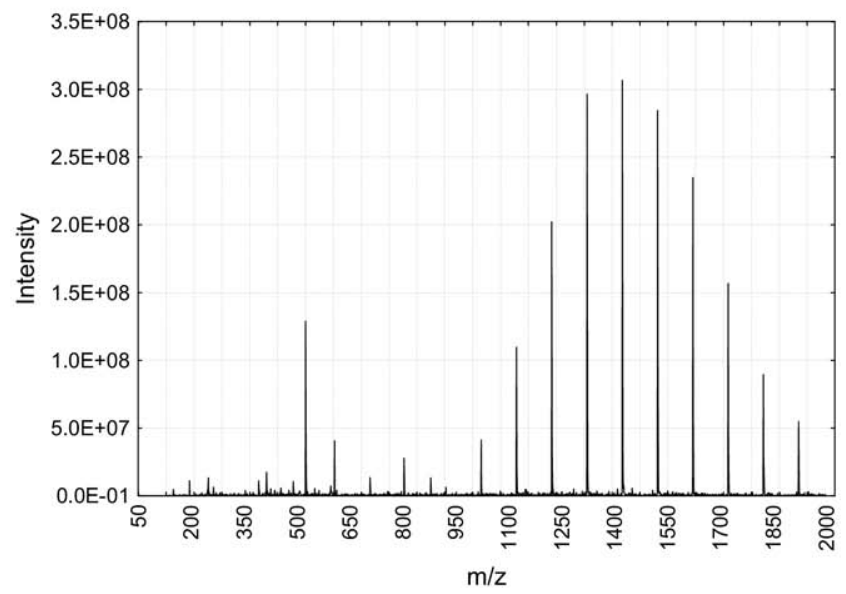

Noise spectrum : $208 \mathrm{~m} / \mathrm{z}$ values

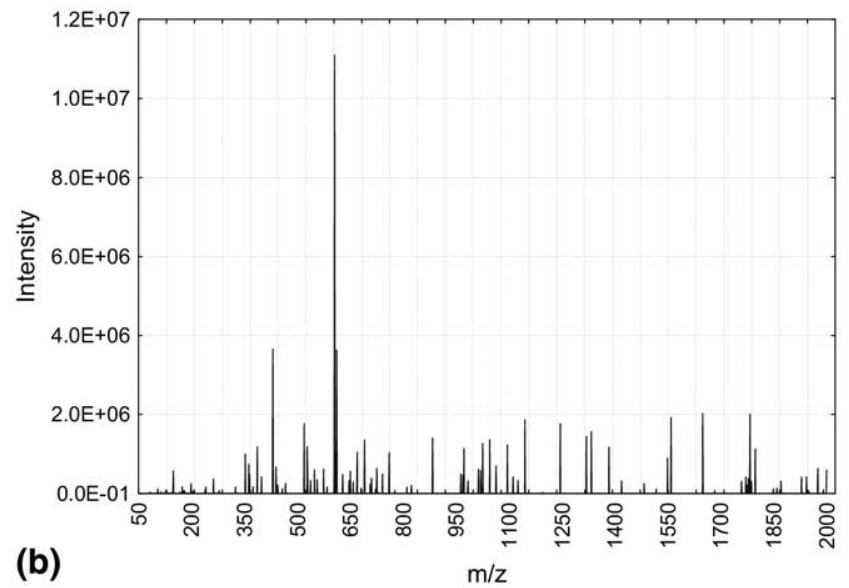

Figure 1. Signal and noise spectra recorded for 1000 scans and: percentage of scans for which the intensities of each $\mathrm{m} / \mathrm{z}$ is different from zero, equal to $100 \%$ (a) and $85 \%$ (b). 


\section{Sum of signal and noise}

$100 \%$

Signal

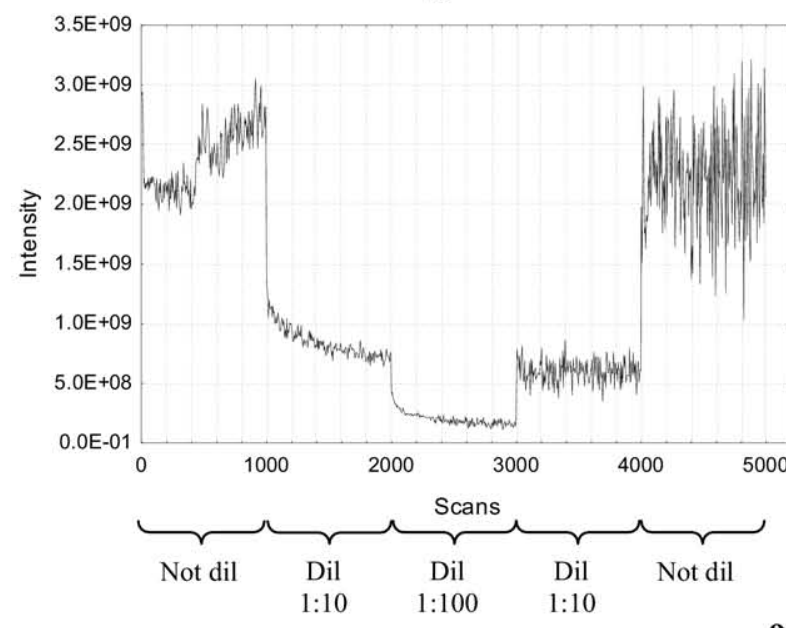

$90 \%$

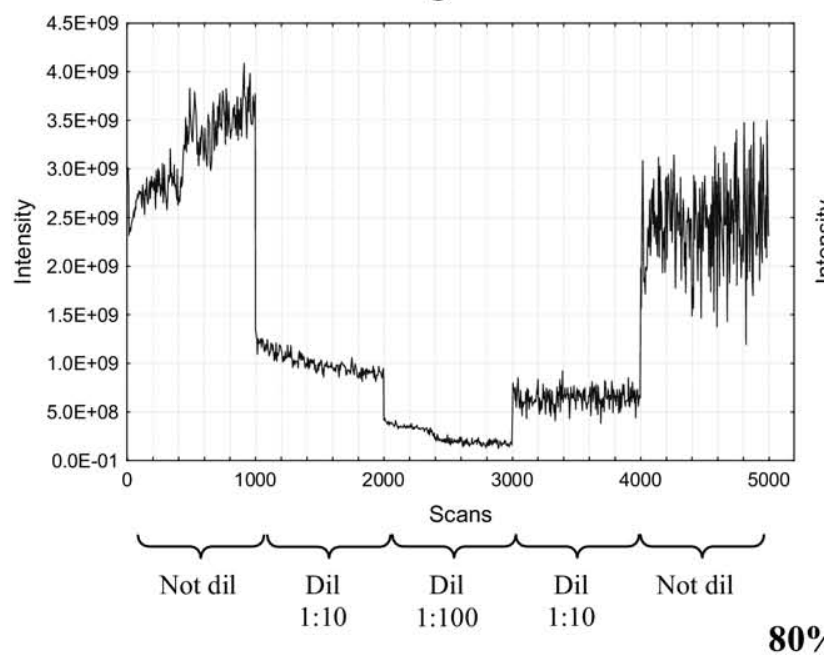

Signal

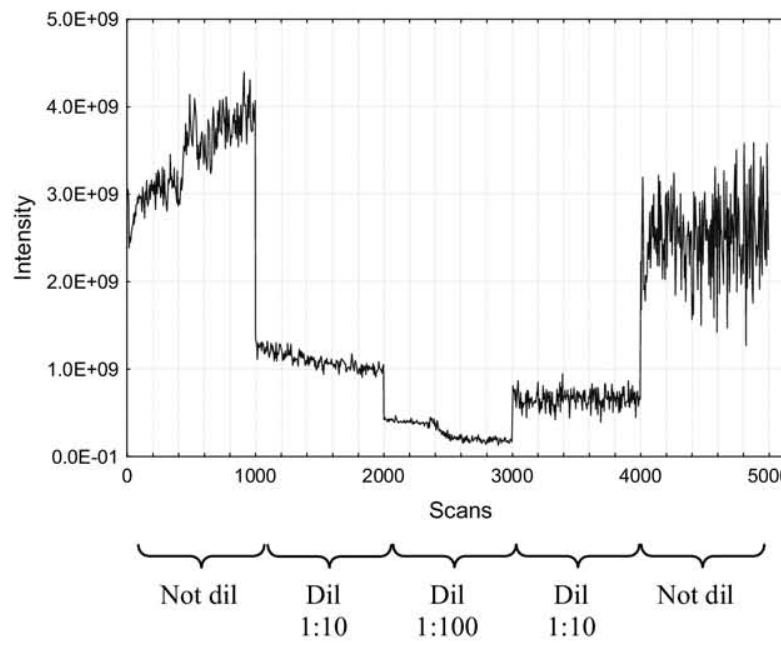

Noise

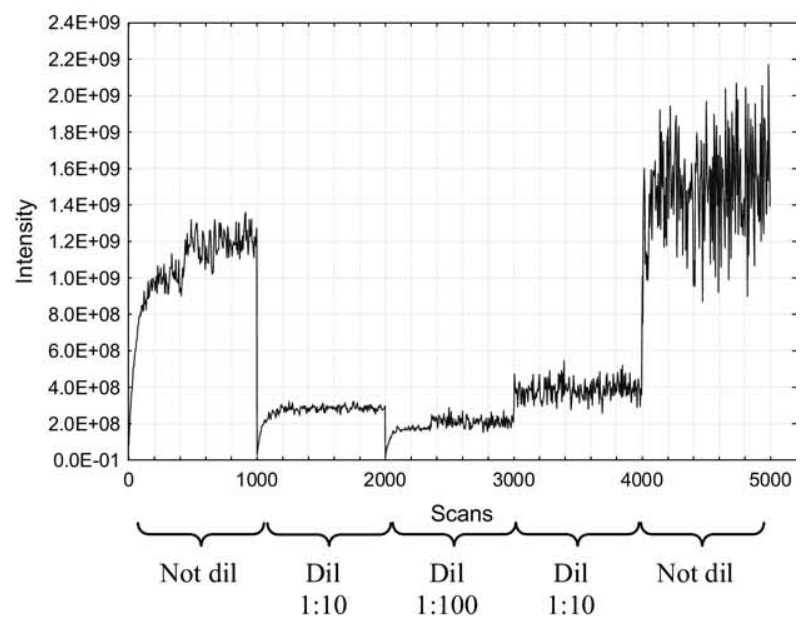

Noise

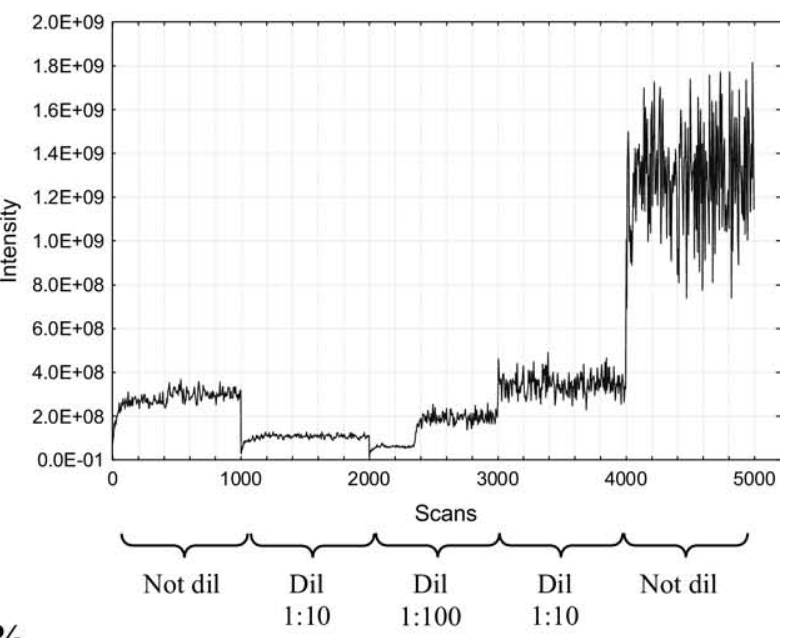

Noise

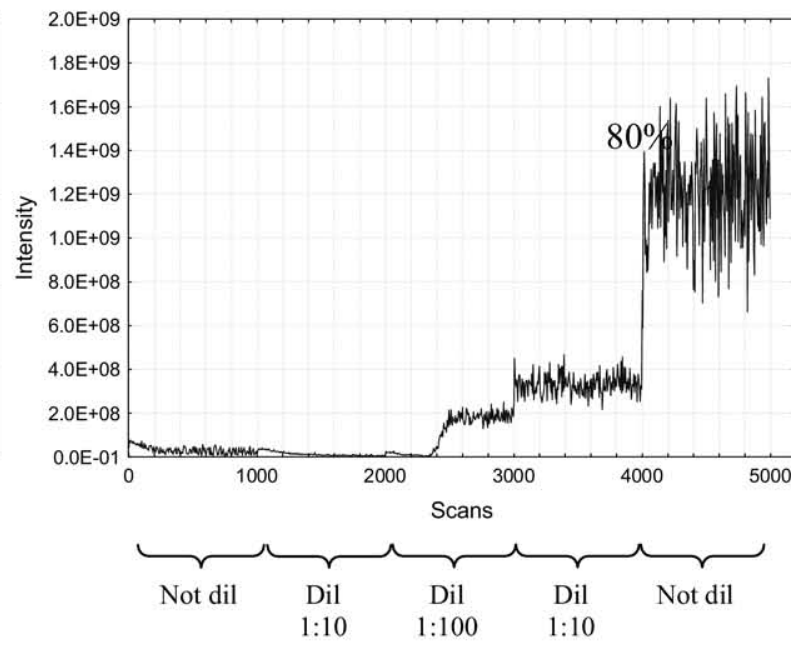

Figure 2. Sum of signal and noise as a function of scans and \% adopted considering 1000 scans for each concentration level of the sequence recorded at decreasing and increasing concentration (the number of scans is reported on the x-axis). 
percentage of scans, the corresponding $m / z$ value was considered as signal; in the opposite case, it was considered as noise. The percentage adopted was varied from 100 to 75 (step 5) and the number of scans from 1000 to 10 (step 10). The variation applied to the percentage adopted allows to explore different standard procedures based on a larger (small \%) or smaller (large \%) pool of selected channels. So, for each pair of parameters two spectra (one for signal and one for noise) are obtained. As index of the overall signal or noise, sum and average value of both spectra were used to provide an estimate of total and average signal and noise. For brevity, only considerations about the sum will be drawn here, since the average value provides similar results.

Figure 1 reports the spectra of signal and noise for 1000 scans if the percentage is varied from 100\% (Figure 1a) to $85 \%$ (Figure $1 \mathrm{~b}$ ); decreasing the percentage the signal spectrum becomes more complex (larger number of channels) and progressively enriches in $\mathrm{m} / \mathrm{z}$ values with small intensities.

The sum of signal and noise varying the number of scans and the percentage selected (surface plot provided as Supplementary Material) shows an increase with the number of scans but a stable plateau is soon reached at $85 \%-75 \%$; the sum of noise increases with the number of scans and shows a rapid decrease at $85 \%$; it seems that the index chosen (sum of both signal and noise) can be used for identifying signal and noise in a complex mass spectrum, in particular at about $85 \%$.

The index chosen has to be tested when a discontinuity is present in the instrumental functioning and the concentration of the standard is varied; therefore, the same calculations were applied to the sequence recorded at different concentration levels. Calculations were performed, varying number and percentage of scans considered at each concentration level independently.

The results with regards to the sum of signal and noise are reported in Figure 2; each graphic shows the sum of signal or noise for all concentration levels in sequence, at three percentages, from $100 \%$ to $80 \%$. Considering the signal calculated at $100 \%$, a clear correspondence with concentration is evident; the discontinuity becomes more evident starting from a percentage of about $90 \%-80 \%$. The sum of the signal at $100 \%$ could be used as target function but in the same situation the noise still contains information on concentration (i.e., signal); if this information is progressively removed from noise and considered as signal (decreasing the percentage), the noise becomes stable before the discontinuity, but a stable noise after the discontinuity is not even reached at $80 \%$. Sum cannot be used as target function since the calculation of $\mathrm{S} / \mathrm{N}$ based on this index is not robust if a discontinuity is present; it would provide a $\mathrm{S} / \mathrm{N}$ not varying with concentration.

It is important to emphasize again that this method is similar to those actually used by standard automatic tuning procedures: if a discontinuity is present, the operator cannot recognize it and the tuning would fail.

\section{Second Method: Principal Component Analysis}

The second method is based on the use of PCA. PCA without centering or autoscaling was first performed separately on three replicates of 1000 scans of full scan mass spectra recorded at the same concentration level of the standard mixture. One replicate was collected before and two replicates after the discontinuity in the instrumental functioning. The results are reported in Table 1. The first PC explains from $99 \%$ to about $97 \%$ of the overall information. Noise, explained by the residuals, accounts therefore for about $1 \%$ before the discontinuity and about 3\% after.

The loadings show a sort of average mass spectrum confirming that $\mathrm{PC}_{1}$ accounts for the average signal (loading plot provided as Supplementary Material). Figure 3a represents the score plot of the first PC; the scores are represented on the $y$-axis while the 1000 scans recorded for each replicate are along the x-axis; the scores of the three replicates are reported in sequence. The scores of the first PC represent the average signal and appear stable with regards to the scale order; the effect of the discontinuity is evident if the general trend is evaluated. The first replicate was carried out when a large autocorrelation is present in the data, reflected in the continuous increasing trend of the scores, while the other two replicates showed a small autocorrelation, reflected in the random variations of the scores. Figure $3 b$ reports instead the $\mathrm{S} / \mathrm{N}$ calculated as the ratio between the score of the first PC (signal) and the standard deviation of the residuals (noise). $\mathrm{S} / \mathrm{N}$ is reported for the three replicates in sequence. This parameter showed a large value with a quite large variation for the first replicate and a more stable trend for the last two replicates. The first PC, giving a score independent on the presence of discontinuities for what concerns its scale order, could be used as target function, while the $\mathrm{S} / \mathrm{N}$ calculated could be used, together with the autocorrelation, as an index, accounting for the presence of discontinuities in the system functioning. They are in facts reflected by changes in the autocorrelation function and in the $\mathrm{S} / \mathrm{N}$ value calculated.

The first PC can be effectively used as target function if the scores of the first PC maintain a trend with the concentration level. PCA was therefore applied to the sequence at variable concentration levels. A separate PCA was carried out for each set of 1000 scans at each

Table 1. Percentage of variance explained by the first three PCs calculated for the three replicates considered

\begin{tabular}{lccc}
\hline & \multicolumn{3}{c}{ \% Explained variance } \\
\cline { 2 - 4 } & ${\text { Replicate } 1^{\mathrm{a}}}$ & Replicate 2 $^{\mathrm{b}}$ & Replicate 3 $^{\mathrm{b}}$ \\
\hline \hline $\mathrm{PC}_{1}$ & 99.56 & 97.22 & 97.21 \\
$\mathrm{PC}_{2}$ & 0.10 & 0.29 & 0.26 \\
$\mathrm{PC}_{3}$ & 0.07 & 0.19 & 0.18 \\
\hline
\end{tabular}

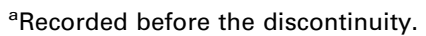

${ }^{b}$ Recorded after the discontinuity. 

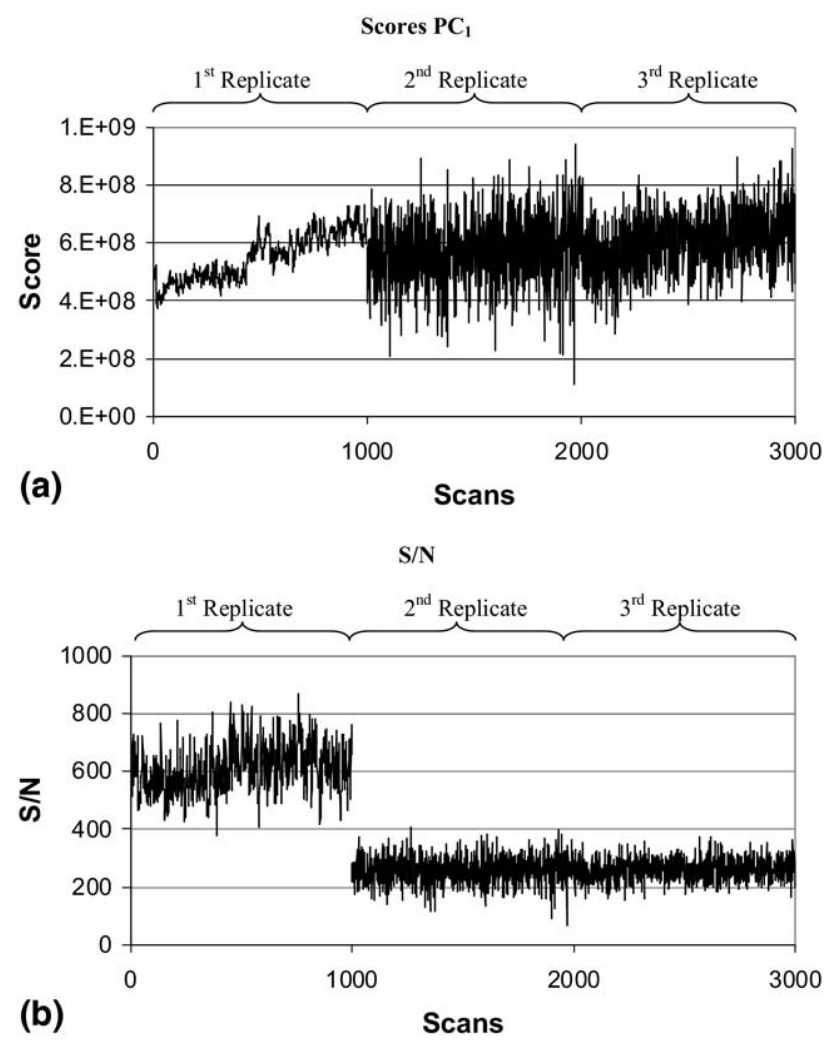

Figure 3. Results for PCA applied on the three genuine replicates of 1000 scans of the calibration mixture not diluted: score plot of the first PC (a); $\mathrm{S} / \mathrm{N}(\mathbf{b})$.

concentration level without centering or autoscaling. The results are reported in Table 2, The first PC explains from $93 \%$ to above $99 \%$ of the overall information.

In the score plot of the first PC (Figure 4a), the scores are reported in sequence for the different concentration levels examined. The score plot indicates that there is correspondence between the scores on the first PC and the concentration level: the scale order of the scores at the same concentration level before and after the discontinuity is comparable. Again, the scores reflect the presence of the discontinuity: they appear characterized by a large variability after the discontinuity, when the autocorrelation function drops. The corresponding behavior of $S / N$ is reported in Figure $4 \mathrm{~b}$. The $S / N$ value shows a variable trend before the discontinuity, while it becomes more stable at lower values after the discontinuity. From these considerations, we can state that the first PC calculated could be effectively used as target

Table 2. Percentage of variance explained by the first PC calculated for each concentration level adopted

$\%$ Variance explained by $\mathrm{PC}_{1}$

\begin{tabular}{ll}
\hline \hline Not diluted & 99.46 \\
$1: 10 \mathrm{vol} / \mathrm{vol}$ & 99.39 \\
$1: 100 \mathrm{vol} / \mathrm{vol}$ & 93.67 \\
$1: 10 \mathrm{vol} / \mathrm{vol}$ & 96.34 \\
Not diluted & 97.22 \\
\hline
\end{tabular}
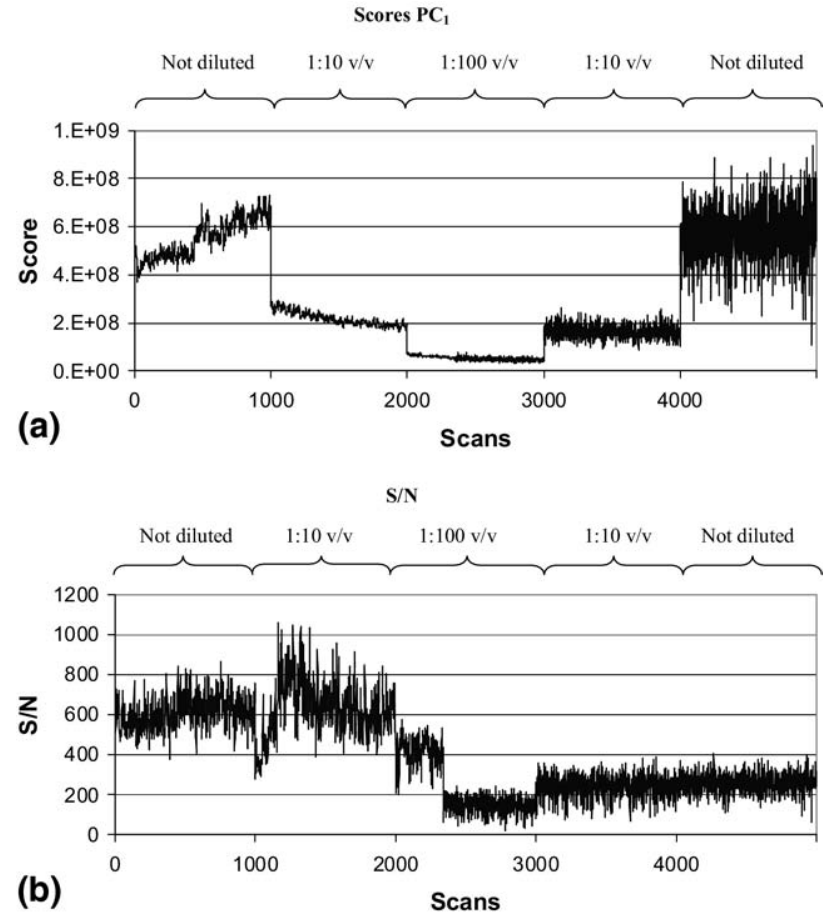

Figure 4. Results for PCA applied on the sequence at different concentration levels of the calibration mixture: score plot of the first PC (a); S/N (b).

function during the phase of tuning of the instrument. The $\mathrm{S} / \mathrm{N}$ thus calculated allows the identification of sudden changes of the system behavior, and can be used as index of bad or good instrumental functioning. With low autocorrelation values (independent subsequent scans) the value of $\mathrm{S} / \mathrm{N}$ calculated by PCA rapidly stabilizes. A number of scans ranging from 10 to 50 is therefore suitable for the application of this method, making it compatible with daily lab practice. Moreover, PCA and the calculation of S/N based on PCA can be easily implemented in the software and automatically managed by the instrument interface.

We think that the use of a lower concentrated standard would be of use during the tuning phase, to avoid saturation effects possibly causing the identified discontinuities.

\section{Appendix A Supplementary Material}

Supplementary material associated with this article may be found in the online version at doi:10.1016/ j.jasms.2009.06.011.

\section{References}

1. March, R. E. An Introduction to Quadrupole Ion Trap Mass Spectrometry. J. Mass Spectrom. 1997, 32, 351-369.

2. Reemtsma, T. The Use of Liquid Chromatography-Atmospheric Pressure Ionization-Mass Spectrometry in Water Analysis, Part I: Achievements. Trends Anal. Chem. 2001, 20, 500-517. 
3. Rosenberg, E. The Potential of Organic (Electrospray and Atmospheric Pressure Chemical Ionization) Mass Spectrometric Techniques Coupled to Liquid-Phase Separation for Speciation Analysis. J. Chromatogr. A 2003, 1000, 841-889.

4. Wong, P. S. H.; Cooks, R. G. Ion Trap Mass Spectrometry. Curr. Sep. $1997,16,85-92$.

5. Finnigan LCQDuo-Getting Started Guide, Revision A. ThermoQuest: 1999.

6. MSD Analytical Technologies Analyst Software-Getting Started Guide. Applied Biosystems: 2008

7. Millennium32 ZQ Mass Detector Software-Getting Started Guide. Waters: 2001.

8. Rogatsky E.; Stein, D. Automated Optimization of Mass Spectrometric Parameters in Tune Mode for Impure Analytes in Dirty Samples Using
Gradient High-Performance Liquid Chromatography. Rapid Commun. Mass Spectrom. 2005, 19, 3759-3760.

9. Moini, M. Ultramark-1621 as a Calibration Reference Compound for Mass-Spectrometry. 2. Positive-Ion and Negative-Ion ElectrosprayIonization. Rapid Commun. Mass Spectrom. 1994, 8, 711-714.

10. Moini, M.; Jones, B. L.; Rogers, R. M.; Jiang, L. Sodium Trifluoroacetate as a Tune/Calibration Compound for Positive and Negative-Ion Electrospray Ionization Mass Spectrometry in the Mass Range of 100-4000 Da. J. Am. Soc. Mass Spectrom. 1998, 9, 977-980.

11. Massart, D. L.; Vanderginste, B. G. M.; Deming, S. M. Michotte, Y. Kaufman, L. Chemometrics: A Textbook; Elsevier: Amsterdam, 1988.

12. Vanderginste, B. G. M.; Massart, D. L.; Buydens, L. M. C.; De Jong, S. Lewi, P. J.; Smeyers-Verbeke, J. Handbook of Chemometrics and Qualimetrics: Part A; Elsevier: Amsterdam, 1998. 